\title{
Cholesterol and saturated fat intake determine the effect of polymorphisms at ABCG5/ABCG8 genes on lipid levels in children
}

Enrique Viturro, $P h D^{1}$, Manuel de Oya, PhD, $M D^{1}$, Miguel A. Lasunción, PhD ${ }^{2}$, Lydia Gorgojo, PhD, $M D^{3}$, José M. Martín Moreno, $\mathrm{PhD}, \mathrm{MD}^{3}$, Mercedes Benavente, $P h D^{1}$, Beatriz Cano, $P h D^{1}$, and Carmen Garces, $P h D^{1}$

Purpose: Analysis of mutations in genes of the cholesterol metabolic pathway has not completely explained the interindividual variability of blood cholesterol concentrations attributed to gene-nutrient interactions. Thus, we analyzed polymorphisms in the ABCG5 and ABCG8 genes, involved in the regulation of intestinal cholesterol absorption, with special interest in a potential interaction with diet to determine lipid levels. Methods: The polymorphisms ABCG5 C1950G (GIn604Glu) and ABCG8 C1895T (Ala640Val) were determined by polymerase chain reaction and restriction analysis in 1227 healthy school children, aged 6 to 8 years. Results: No significant differences were found in blood lipid levels between subjects with different genotypes of the two analyzed polymorphisms. However, important differences appeared when separating subjects by their different lipid intake. The presence of the ABCG8 C1895T and ABCG5 C1950G polymorphisms was associated with different plasma total cholesterol, low-density lipoprotein cholesterol complex, and apolipoprotein B levels only in low-cholesterol consumers (significantly for the C1895T polymorphism), and among children within the lower tertile of saturated fat intake (significantly for the C1950G polymorphism). Conclusion: Polymorphisms at the half-transporter ABCG5 and $A B C G 8$ genes affect blood cholesterol concentrations in prepubertal children by influencing dietary responsiveness. This highly significant gene-nutrient interaction could explain the great individual differences in the plasma lipid response to cholesterol and fat intake. Genet Med 2006:8(9):594-599.

Atherosclerosis is a complex, multifactorial disease in which many genes, environmental factors, and interactions between them are involved. Increased consumption of cholesterol and saturated fat has been shown to be associated with higher plasma cholesterol levels and increased risk of cardiovascular disease. ${ }^{1,2}$ However, well-controlled experimental studies have demonstrated that individuals differ widely in the response of their plasma cholesterol concentrations to dietary cholesterol and saturated fat. ${ }^{3,4}$ A genetic determination of this variability has been documented;5,6 however, the analysis of the influence of common mutations in relevant genes of cholesterol metabolic pathways (apolipoproteins [apo], receptors, and enzymes) on the responsiveness to dietary fat and cholesterol has not completely explained the wide interindividual variability in the responsiveness to diet, ${ }^{7}$ suggesting the intervention of other

\footnotetext{
From the ${ }^{1}$ Lipid Unit, Fundación Jiménez Díaz, Universidad Autónoma de Madrid, Madrid, Spain; ${ }^{2}$ Biochemistry Research Department, Hospital Ramón y Cajal, Universidad de Alcalá, Madrid, Spain; ${ }^{3}$ Department of Preventive Medicine, Universidad de Valencia, Valencia, Spain.

Carmen Garcés, PhD, Unidad de Lípidos, Fundación Jiménez Díaz Avda, Reyes Católicos, 2 28040 Madrid, Spain.

Submitted for publication March 29, 2006.

Accepted for publication June 26, 2006.

DOI: 10.1097/01.gim.0000237760.25195.e7
}

unknown genes and factors. Studies undertaken to determine the relationship between dietary cholesterol absorption and plasma lipoprotein levels showed large individual differences in dietary cholesterol absorption, suggesting a genetic variation among humans in the regulation of this process. ${ }^{8,9}$

When cholesterol absorption was studied, two members of the human adenosine triphosphate (ATP) Binding Cassette $(A B C)$ transporter family, ${ }^{10} A B C G 5$ and $A B C G 8$, seem to play a capital role. ${ }^{10,11}$ These two ATP-dependent half-transporters join to form a transport unit that regulates the absorption of cholesterol from the diet (intestine) and its excretion in the bile (liver). ${ }^{11}$ Their discovery came from the study of a rare genetic disease, sitosterolemia, characterized by abnormal sterol levels in the blood, caused by the genetic disruption of the ABCG5/G8 transport unit. ${ }^{12}$ Several mutations in the ABCG5 and ABCG8 genes were identified at this time in sitosterolemic patients. ${ }^{13-15}$ In addition, common polymorphic variants of these genes have been hypothesized to be related to plasma lipid level differences in the general population, although this association is not clear yet. ${ }^{16,17}$ Thus, in this study we examined the influence of two of those polymorphisms in the ABCG5 and ABCG8 genes (C1950G [Gln604Glu] and C1895T [Ala640Val], respectively), on determining plasma lipid levels in a sample-based population of 1227 healthy Spanish children, with special attention to the potential effect of dietetic parameters in this determination. 


\section{MATERIALS AND METHODS}

\section{Population}

The study population included 1227 healthy school children (621 males and 606 females) between 6 and 8 years old (average age of 6.7 years) who participated in a voluntary analysis of cardiovascular risk factors in Spain over the period 1998 to 2000. All were free of any endocrine, metabolic, hepatic, or renal disorder. Sampling was randomized and stratified by pools of school centers in each participating city. More detailed information about the design of the study is available in previous publications. ${ }^{18,19}$ The study protocol complied with Helsinki Declaration guidelines and Spanish legal provisions governing clinical research on humans, and was approved by the Clinical Research Ethics Committee of the Fundación Jiménez Díaz in Madrid, Spain. Parents were required to sign a written consent for participation of their children in the study.

\section{Anthropometric measurements}

Height and weight were determined when children were lightly dressed and without shoes. Height was measured to the nearest $0.1 \mathrm{~cm}$ using a portable stadiometer, and weight was recorded to the nearest $0.1 \mathrm{~kg}$ using a standardized electronic digital scale. From these measurements, a body mass index (weight [kilograms] divided by the square of height [meter]) was calculated.

\section{Blood sampling, lipid, and apolipoprotein analyses}

Fasting (12 hours) venous blood samples were obtained from every child early in the morning by venipuncture into a Vacutainer tube containing EDTA. Plasma cholesterol and triglyceride levels were determined enzymatically (Menarini Diagnostics, Florence, Italy) with an RA-1000 Autoanalyzer. High-density lipoprotein-cholesterol complex (HDL-C) was measured after precipitation of apoB-containing lipoproteins with phosphotungstic acid and magnesium (Roche Diagnostics, Basel, Switzerland). Low-density lipoprotein-cholesterol (LDL-C) was calculated according to Friedewald's formula. Plasma apoAI and apoB concentrations were quantified by immunonephelometry (Array System, Beckman Coulter, Fullerton, CA).

Analytic variability was evaluated by Quality Control Service (Roche) using commercial control sera (Precinorm and Precipath, Roche). The interassay coefficients of variation were as follows: cholesterol $1.4 \%$, triglyceride $1.7 \%$, apoAI $5.5 \%$, and apoB $4.8 \%$.

\section{DNA extraction and polymorphism analysis}

Genomic DNA was prepared from leukocytes. For ABCG5 C1950G genotyping, DNA was amplified by polymerase chain reaction using the primers 5' CCTTGACAGGCAGCTCAAATG $3^{\prime}$ and $5^{\prime}$ CAGAGCCAGTCATGCACAGTCG 3' as described. ${ }^{13}$ The 260-base pair amplified fragment was restricted with the enzyme PdmI, and the DNA fragments were separated by an $8 \%$ polyacrylamide gel electrophoresis. For ABCG8 C1895T genotyping, DNA was amplified by polymerase chain reaction using the primers 5' ATGAGCCCACTGCATGTCT 3' and 5' AGTTGAAGGGTCTGCTCAGGT 3', and the 219-base pair amplified fragment was restricted with the enzyme NcoI. ${ }^{13}$

\section{Nutritional data}

Information on food and nutrition was obtained through a food frequency questionnaire (FFQ). A complete description of the FFQ is available in previous publications. ${ }^{19,20}$ Food and nutritional data were collected, at each school, by a field team made up of a physician, a nurse, and a group of persons purpose-trained in the use of this FFQ. The questionnaire included a total of 77 food codes grouped under 11 heads by affinity in nutrient content. For each food, the usual size of the serving eaten was defined and the mean frequency of consumption of such servings over the previous year was ascertained. With the use of Spanish food-composition tables, a food frequency conversion program was designed that furnished a database with the annual food consumption and daily nutrient intake frequencies for each individual surveyed. This enabled nutrient and total caloric intake to be estimated.

\section{Statistical analysis}

Statistical analyses were carried out with the SPSS software package, version 9.0 (SPSS Inc., Chicago, IL). The Student $t$ and chi-square tests were applied to test differences in means and percentages between boys and girls and between groups consuming more or less than $300 \mathrm{mg} /$ day of cholesterol. A $t$ test analysis was used to compare lipid and apolipoprotein levels between subjects with or without the mutations.

\section{RESULTS}

Table 1 shows anthropometric, biochemical, and genetic data from the children according to gender. The frequencies of the ABCG8 C1895T genotypes were $63.2 \%$ for AA, $33.1 \%$ for $\mathrm{AV}$, and $3.7 \%$ for VV. The prevalence of the V allele was $20.2 \%$. The frequencies of the ABCG5 C1950G genotypes were $71.8 \%$ for CC, $27.1 \%$ for CG, and only $1.1 \%$ for GG. The prevalence of the $\mathrm{G}$ allele was $14.6 \%$; $54.9 \%$ of the children in our study present the mutation in at least one of the genes. Genotype frequencies did not deviate from the Hardy-Weinberg equilibrium.

Table 2 shows mean plasma lipid levels by gender according to the ABCG8 C1895T and ABCG5 C1950G variations. We did not find any significant differences in plasma lipid levels between subjects with the different genotypes of either of the two polymorphisms in either sex.

Influence of diet in the effect of these polymorphisms on blood lipid levels was also to be tested. We examined the influence of the polymorphisms on lipid levels according to adherence to the World Health Organization's recommendations for cholesterol intake ( $<300 \mathrm{mg} /$ day). Mean daily cholesterol intake was $244 \mathrm{mg} /$ day in those with a cholesterol intake less than $300 \mathrm{mg} /$ day and $422 \mathrm{mg} /$ day in children with a cholesterol intake of $300 \mathrm{mg} /$ day or greater. The mean caloric intake was $1895 \mathrm{kcal} /$ day in the low cholesterol intake group and 2272 $\mathrm{kcal} /$ day in the high cholesterol intake group. Except for apoAI 
Table 1

Anthropometric, biochemical, and genotypic characteristics from children according to gender

\begin{tabular}{|c|c|c|c|c|}
\hline & & $\begin{array}{c}\text { Boys } \\
(\mathrm{n}=621)\end{array}$ & $\begin{array}{c}\text { Girls } \\
(\mathrm{n}=607)\end{array}$ & $\begin{array}{c}\text { Total } \\
(\mathrm{n}=1228)\end{array}$ \\
\hline Age $(y)$ & & $6.70(0.69)$ & $6.70(0.70)$ & $6.71(0.70)$ \\
\hline Weight $(\mathrm{kg})$ & & $26.95(5.31)$ & $26.70(5.47)$ & $26.83(5.39)$ \\
\hline Height (m) & & $1.26(0.06)$ & $1.25(0.07)^{a}$ & $1.25(0.06)$ \\
\hline BMI $\left(\mathrm{kg} / \mathrm{m}^{2}\right)$ & & $16.98(2.40)$ & $17.00(2.51)$ & $16.99(2.45)$ \\
\hline $\mathrm{TC}(\mathrm{mg} / \mathrm{dL})$ & & $181.7(26.0)$ & $183.8(28.1)$ & $182.8(27.9)$ \\
\hline LDL-C (mg/dL) & & $107.4(24.9)$ & $110.3(26.2)^{a}$ & $108.9(25.6)$ \\
\hline apoB $(\mathrm{mg} / \mathrm{dL})$ & & $68.9(14.1)$ & $71.5(14.8)^{b}$ & $70.2(14.5)$ \\
\hline $\mathrm{TG}(\mathrm{mg} / \mathrm{dL})$ & & $70.3(22.5)$ & $73.2(24.9)^{a}$ & $71.7(23.7)$ \\
\hline HDL-C (mg/dL) & & $60.2(13.0)$ & $58.9(13.1)$ & $59.5(13.1)$ \\
\hline apoAI (mg/dL) & & $138.2(18.9)$ & $135.7(18.8)^{a}$ & $137.0(18.9)$ \\
\hline \multirow{3}{*}{$\begin{array}{l}\text { ABCG8 C1895T } \\
\text { genotype }\end{array}$} & $\mathrm{AA}$ & $382(61.5)$ & $395(65.0)$ & $777(63.2)$ \\
\hline & AV & $216(34.8)$ & $190(31.4)$ & $406(33.1)$ \\
\hline & VV & $23(3.7)$ & $22(3.6)$ & $45(3.7)$ \\
\hline \multirow{3}{*}{$\begin{array}{l}\text { ABCG5 C1950G } \\
\text { genotype }\end{array}$} & CC & $452(72.8)$ & $429(70.8)$ & $881(71.8)$ \\
\hline & CG & $164(26.4)$ & $168(27.7)$ & $332(27.1)$ \\
\hline & GG & $5(0.8)$ & $10(1.5)$ & $15(1.1)$ \\
\hline
\end{tabular}

BMI, body mass index; TC, total cholesterol; TG, triglyceride; LDL-C, lowdensity lipoprotein-cholesterol; apo, apolipoprotein; HDL-C, high-density lipoprotein-cholesterol.

Quantitative values are listed as mean (standard deviation) and genotype frequencies as $\mathrm{n}(\%)$.

${ }^{a}$ Significantly different from boys $(P<.05)$.

${ }^{b}$ Significantly different from boys $(P<.01)$. levels (significantly higher in those children with a cholesterol intake $\geq 300 \mathrm{mg} /$ day), cholesterol intake did not affect lipid levels in the group as a whole (data not shown). However, when analyzing the influence on lipid levels of the ABCG5 C1950G and ABCG8 C1895T polymorphisms in noncarriers of the other studied mutation, depending on the intake of dietary cholesterol, we found that total cholesterol (TC) and LDL-C and apoB concentrations were different by genotypes for both polymorphisms only among those children consuming lower amounts of cholesterol. These differences were significant for the $A B C G 8$ C1895T polymorphism in the group of subjects without the ABCG5 1950G allele in boys (Table 3) and in girls (Table 4). Among those consuming more than the recommended $300 \mathrm{mg} /$ day of cholesterol, no differences were found between genotypes. In girls, the presence of the ABCG8 AV genotype was associated with significantly higher TC $(16.9 \mathrm{mg} /$ $\mathrm{dL} ; P<.01)$, LDL-C $(14.8 \mathrm{mg} / \mathrm{dL} ; P<.01)$, and apoB $(8.4$ $\mathrm{mg} / \mathrm{dL} ; P<.01$ ) levels when consuming less than $300 \mathrm{mg} /$ day of cholesterol, whereas no significant differences existed with a cholesterol intake over that limit (Table 4). No significant interaction was found between the polymorphisms and cholesterol intake on HDL-C, TG, or apoAI.

When the effect of saturated fat intake was studied, a division of the population according to adherence to the World Health Organization's recommendations was not possible, because only 2 of the more than 1000 children included in the study were found to comply with the recommendations. Therefore, the population was divided in statistical tertiles of saturated fat intake. The mean concentrations of daily saturated fat intake ranged from 14.3 to $34.1 \mathrm{~g} /$ day in tertile I, 34.2 to $41.9 \mathrm{~g} /$ day in tertile II, and 42.0 to $104.4 \mathrm{~g} /$ day in tertile III. When analyzing by tertiles, we found

Table 2

Plasma lipid levels according to the ABCG5 C1950G and ABCG8 C1895T polymorphisms

\begin{tabular}{|c|c|c|c|c|c|c|c|}
\hline & & $\mathrm{TC}$ & LDL-C & ароB & TG & HDL-C & apoAI \\
\hline \multirow[t]{2}{*}{ ABCG8 C1895T } & AA (382) & $181.2(26.6)$ & $107.1(25.3)$ & $68.7(14.2)$ & $70.7(22.2)$ & $60.0(13.1)$ & $137.5(19.1)$ \\
\hline & VV (23) & $178.4(23.5)$ & $102.6(24.5)$ & $68.4(13.4)$ & $65.8(21.9)$ & $62.6(14.6)$ & $142.4(16.8)$ \\
\hline \multirow[t]{2}{*}{ ABCG5 C1950G } & CC (452) & $181.7(25.8)$ & $107.2(25.0)$ & $69.0(14.0)$ & $70.6(23.3)$ & $60.4(12.9)$ & $138.3(18.7)$ \\
\hline & GG (5) & $173.6(38.6)$ & $102.2(27.7)$ & $67.0(14.6)$ & $63.1(7.2)$ & $58.8(12.5)$ & $132.6(10.4)$ \\
\hline \multicolumn{8}{|l|}{ Girls } \\
\hline \multirow[t]{2}{*}{ ABCG8 C1895T } & AA (395) & $182.7(28.0)$ & $109.3(25.6)$ & $71.3(14.6)$ & $72.6(14.6)$ & $58.9(12.8)$ & $135.5(18.7)$ \\
\hline & AV (190) & $186.3(28.4)$ & $112.2(27.4)$ & $72.4(15.4)$ & $74.8(24.1)$ & $59.2(13.5)$ & $136.6(18.8)$ \\
\hline & GG (10) & $171.6(24.1)$ & 94.7 (28.7) & $64.9(9.8)$ & $64.1(12.2)$ & $64.1(22.0)$ & $136.6(18.5)$ \\
\hline
\end{tabular}

TC, total cholesterol; LDL-C, low-density lipoprotein-cholesterol; apo, apolipoprotein; TG, triglyceride; HDL-C, high-density lipoprotein-cholesterol. Values are given as mean (standard deviation), and expressed in milligrams/deciliter. 
Table 3

Plasma lipid levels according to the ABCG5 C1950G and ABCG8 C1895T polymorphisms and cholesterol and saturated fat intake in boys

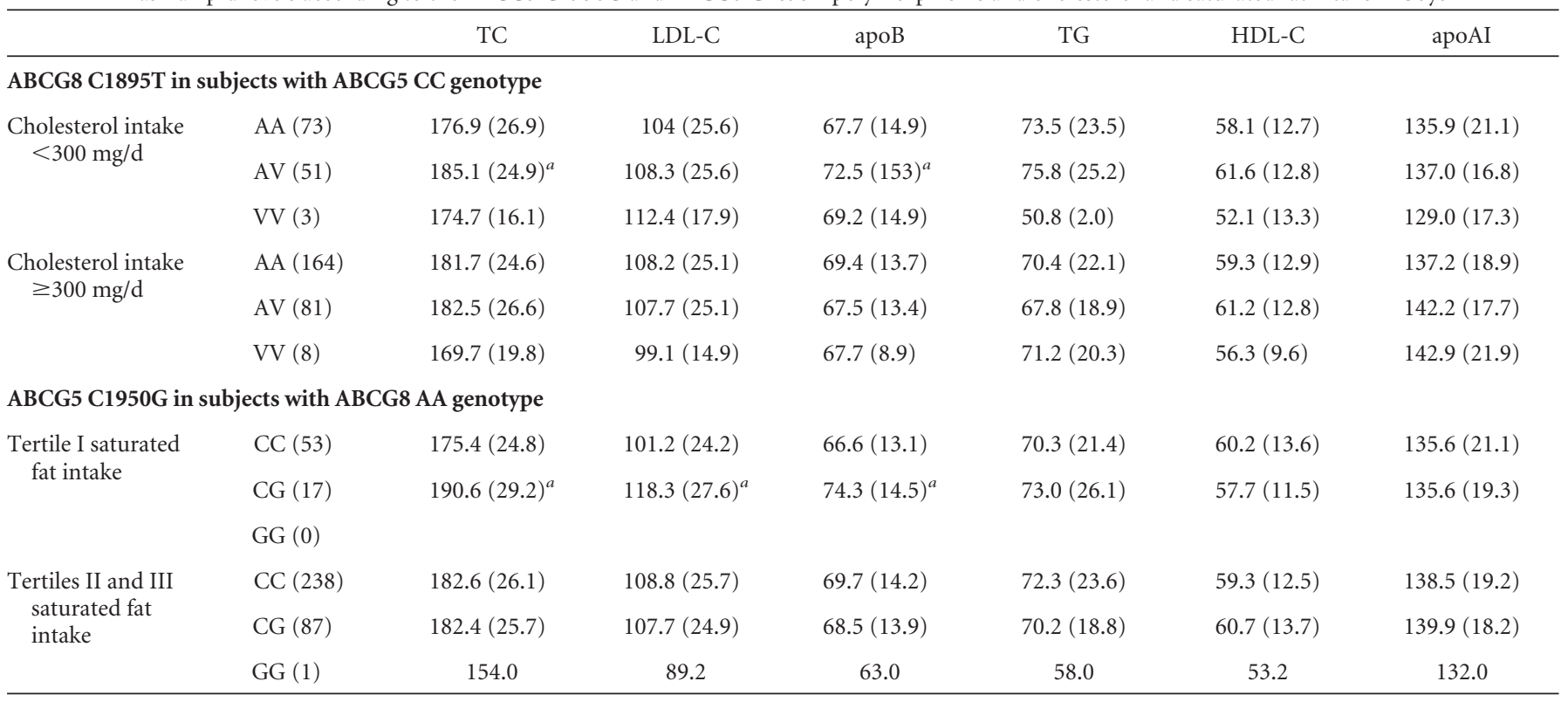

TC, total cholesterol; LDL-C, low-density lipoprotein-cholesterol; apo, apolipoprotein; TG, triglyceride; HDL-C, high-density lipoprotein-cholesterol. Values are given as mean (standard deviation), and expressed in milligrams/deciliter.

${ }^{a}$ Significantly different from noncarriers of the minor allele $(P<.05)$.

Table 4

Plasma lipid levels according to the ABCG5 C1950G and ABCG8 C1895T polymorphisms and cholesterol and saturated fat intake in girls

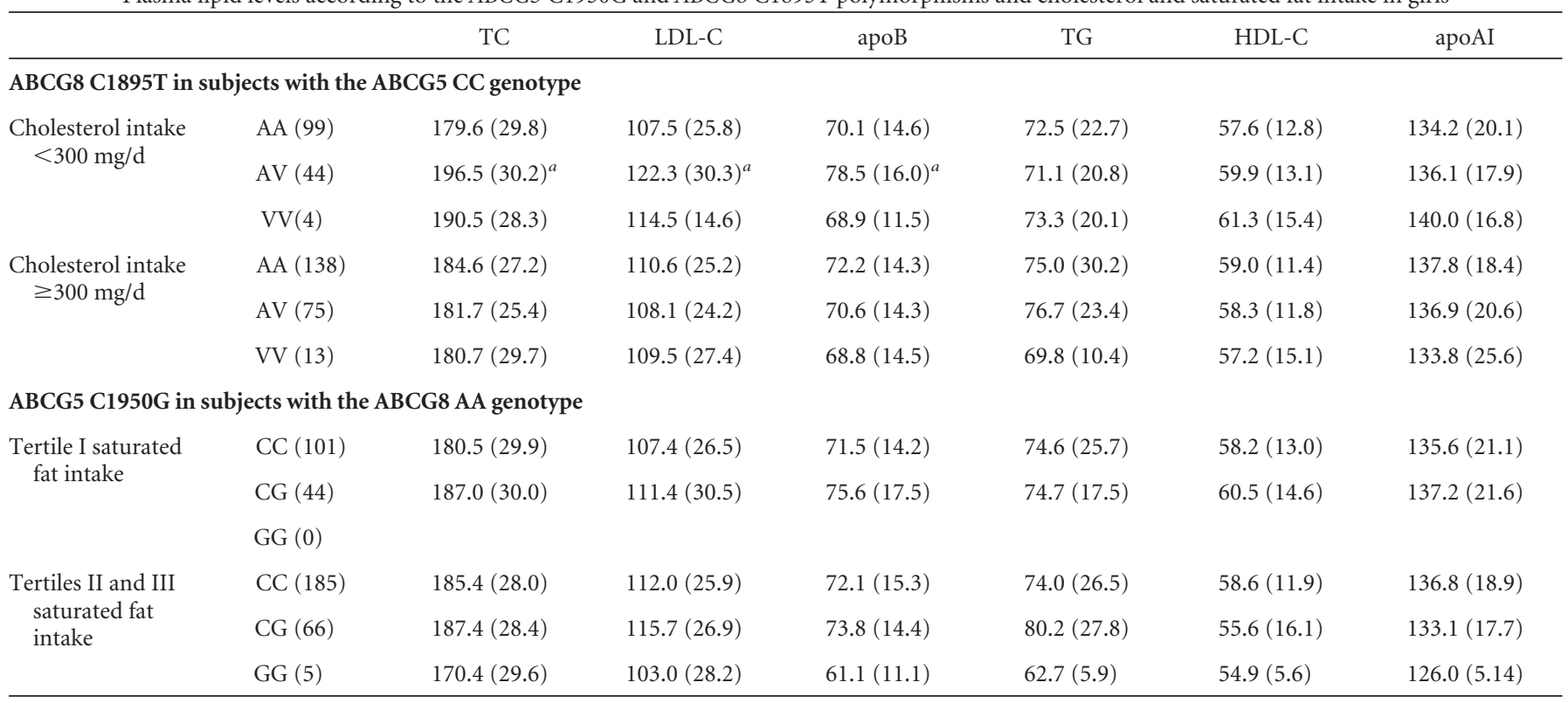

TC, total cholesterol; LDL-C, low-density lipoprotein-cholesterol; apo, apolipoprotein; TG, triglyceride; HDL-C, high-density lipoprotein-cholesterol.

Values are given as mean (standard deviation), and expressed in milligrams/deciliter.

${ }^{a}$ Significantly different from noncarriers of the minor allele $(P<.01)$.

differences between genotypes for the ABCG5 C1950G and ABCG8 C1895T polymorphisms in noncarriers of the minor allele of the other studied polymorphism in boys and in girls only in the low tertile of saturated fat intake (Tables 3 and 4 ). Thus, in this lowest tertile, male noncarriers of the ABCG8 C1895T mutation who were carriers of the minor allele $(\mathrm{G})$ of $A B C G 5 \mathrm{C} 1950 \mathrm{G}$ polymorphism had significantly higher TC $(15.2 \mathrm{mg} / \mathrm{dL} ; P<.05)$, LDL-C $(17.1 \mathrm{mg} / \mathrm{dL} ; P<.05)$, and apoB $(4.1 \mathrm{mg} / \mathrm{dL} ; P<.05)$ levels (Table 3). Levels between groups of children within the highest tertiles of saturated fat intake are almost identical. 


\section{DISCUSSION}

Dietary recommendations are important to achieve controlled lipid levels. However, not all individuals benefit equally from fat and cholesterol intake restriction. ${ }^{3,4}$ Genetic factors seem to be related with whether individuals are either "dietary responsive" or "dietary nonresponsive." 5,6 Mutations in cholesterol metabolic pathways fail to completely explain this genetic determination, ${ }^{7}$ justifying the analysis of mutations in another metabolic process that could influence cholesterol responsiveness: the absorption of dietary cholesterol. A new line of investigation has been opened with the study of the $\mathrm{ABC}$ half-transporters G5 ( $A B C G 5)$ and G8 (ABCG8) that cooperate to provide a barrier to cholesterol accumulation. ${ }^{11}$

On studying the influence of a frequent polymorphism in each one of these genes in a population-based sample of Spanish children, we found a highly significant gene-nutrient interaction on determining lipid levels. The ABCG5 C1950G and $A B C G 8$ C1895T polymorphisms were not related with the lipid profile when analyzing the group as a whole. Diet is not directly related to plasma lipid levels either. However, when high ( $\geq 300 \mathrm{mg} /$ day) and low ( $<300 \mathrm{mg} /$ day) cholesterol intake groups or tertiles of saturated fat intake were considered, an important association of the ABCG8 C1895T and ABCG5 C1950G polymorphisms with blood lipid levels was observed, with significantly higher TC, LDL-C, and apoB levels in carriers of the less common allele for these polymorphisms only among the children consuming less than the recommended limit of $300 \mathrm{mg} /$ day of cholesterol or belonging to the lower tertile of saturated fat intake. Such differences are not present in children consuming more than the recommended limit of cholesterol or in the higher tertile of saturated fat.Other significant gene-nutrient interactions have been reported for fat intake. Fat intake was found to modify the effect of a polymorphism at the hepatic lipase gene on HDL-C levels. ${ }^{21}$ The possible effect of the ABCG5 C1950G polymorphism on responsiveness to diet has been suggested for adults. ${ }^{17}$

Our data showed that consuming less than $300 \mathrm{mg} /$ day of cholesterol or a lower amount of fat was associated with significantly lower TC, LDL-C, and apoB levels only when the studied mutations were not present. Because of the high prevalence of these mutations, these results would explain the observation that in an important percentage of the general population, diets with high cholesterol intake are not always associated with higher TC, LDL-C, and apoB concentrations, and cholesterolrestrictive diets are not always associated with a decrease in blood lipid levels. According to these results, it is currently well documented that although some individuals show a significant response to high dietary cholesterol, with lipid levels increases of more than twofold the mean, others are unresponsive and even have decreasing cholesterol levels. ${ }^{3,22-25}$ To date, studies based on classic genetic analysis have not explained these results, but our findings will contribute to clarify this issue.

In our population, we previously described an important influence of apoE genotype on TC, LDL-C, and apoB levels, ${ }^{18,26}$ but the results reported here remain without signif- icant changes after correcting by this factor (data not shown). The design of our study cannot address the mechanism by which cholesterol or saturated fat interacts with the polymorphisms. A plausible hypothesis for future works could be that because the ABC transporters are ATP dependent, they may present sigmoidal saturation kinetics. Thus, there is a range of low cholesterol intake in which differences between genotypes can be appreciated, and a threshold in which the system is saturated by the substrate, regardless of the genotype, and the differences are no longer apparent. In support of this hypothesis, previous works show that although increasing dietary cholesterol within the physiologic range resulted in overall reductions in the efficiency of cholesterol absorption, this effect was weaker in some individuals and stronger in others. ${ }^{27}$

On the basis of our results, we conclude that the effect of dietary cholesterol and saturated fat on TC, LDL-C, and apoB depends on the two widely existing polymorphisms on the half-transporter ABCG5 and ABCG8 genes. The relevance of the implications of our findings arises from the fact that these mutations are widely present in our population; $54.9 \%$ of the children in our study are carriers of the mutation in at least one gene. This highly significant gene-nutrient interaction helps to explain the large individual differences in the plasma lipid response to cholesterol and saturated fat intake, and why some individuals do not apparently benefit from cholesterol-restrictive diets. Further studies are necessary to determine whether this association is also found in adults and whether other existing polymorphisms in ABCG5 and ABCG8 present similar or different interactions with diet when determining blood lipid levels.

\section{ACKNOWLEDGMENTS}

This study was supported by grants from the Fondo de Investigación Sanitaria FIS 02/3104, 020994 and 02/0241. Viturro, Benavente, and Cano are fellows of the Conchita Rábago Foundation. We thank Alexander G. Borun for revision of the article.

\section{References}

1. Grundy SM, Denke MA. Dietary influences on serum lipids and lipoproteins. J Lipid Res 1990;31:1149-1172.

2. Sonnenberg LM, Posner BM, Belanger AJ, Cupples LA, et al. Dietary predictors of serum cholesterol in men: the Framingham cohort population. J Clin Epidemiol 1992;45:413-418.

3. Katan MB, Beynen AC, de Vries JH, Nobels A. Existence of consistent hypo- and hyper-responders to dietary cholesterol in man. Am J Epidemiol 1986;123:221-234.

4. McNamara DJ, Kolb R, Parker TS, Batwin H, et al. Heterogeneity of cholesterol homeostasis in man. Response to changes in dietary fat quality and cholesterol quality. J Clin Invest 1987;79:1729-1739.

5. Clifton PM, Abbey M. Genetic control of response to dietary fat and cholesterol. World Rev Nutr Diet 1997;80:1-14.

6. Katan MB, Grundy SM, Willett WC. Should a low-fat, high-carbohydrate diet be recommended for everyone? Beyond low-fat diets. N Engl J Med 1997;337:563566.

7. Ye SQ, Kwiterovich PO Jr. Influence of genetic polymorphisms on responsiveness to dietary fat and cholesterol. Am J Clin Nutr 2000;72:1275S-1284S.

8. Sehayek E, Nath C, Heinemann T, McGee M, et al. U-shape relationship between change in dietary cholesterol absorption and plasma lipoprotein responsiveness and evidence for extreme interindividual variation in dietary cholesterol absorption in humans. J Lipid Res 1998;39:2415-2422. 
9. Bosner MS, Lange LG, Stenson WF, Ostland RE Jr. Percent cholesterol absorption in normal women and men quantified with dual stable isotopic tracers and negative ion mass spectrometry. J Lipid Res 1999;40:302-308.

10. Dean M, Hamon Y, Chimini G. The human ATP-binding cassette (ABC) transporter superfamily. J Lipid Res 2001;42:1007-1017.

11. Berge KE, Tian H, Graf GA, Yu L, et al. Accumulation of dietary cholesterol in sitosterolemia caused by mutations in adjacent ABC transporters. Science 2000;290: 1771-1775.

12. Yu L, Hammer RE, Li-Hawkins J, Von Bergmann K, et al. Disruption of Abcg5 and Abcg8 in mice reveals their crucial role in biliary cholesterol secretion. Proc Nat Acad Sci U S A 2002;99:16237-16242.

13. Hubacek JA, Berge KA, Cohen JC, Hobbs HH. Mutations in ATP-binding cassette proteins G5 (ABCG5) and G8 (ABCG8) causing sitosterolemia. Hum Mutat 2001; 18:359-360.

14. Heimerl S, Langmann T, Moehle C, Mauerer R, et al. Mutations in the human ATPbinding cassette transporters ABCG5 and ABCG8 in sitosterolemia. Hum Mutat 2002;20:151.

15. Lu K, Lee M-H, Hazard S, Brooks-Wilson A, et al. Two genes that map to the STSL locus cause sitosterolemia: genomic structure and spectrum of mutations involving sterolin-1 and sterolin-2, encoded by ABCG5 and ABCG8, respectively. Am J Hum Genet 2001;69:278-290.

16. Berge KE, von Bergmann K, Lutjohann D, Guerra R, et al. Heritability of plasma noncholesterol sterols and relationship to DNA sequence polymorphism in ABCG5 and ABCG8. J Lipid Res 2002;43:486-494.

17. Weggemans RM, Zock PL, Tai ES, Ordovas JM, et al. ATP-binding cassette G5 C1950G polymorphism may affect blood cholesterol concentration in humans. Clin Genet 2002;62:226-229.
18. Garcés $\mathrm{C}$, Benavente M, Ortega $\mathrm{H}$, Rubio R, et al. Influence of birth weight on the Apo $\mathrm{E}$ genetic determinants of plasma lipid levels in children. Pediatr Res 2002;52:873-878.

19. Royo-Bordonada MA, Gorgojo L, de Oya M, Garces C, et al. Food sources of nutrients in the diet of Spanish children: The Four Provinces Study. Br J Nutr 2003;89:105-114.

20. Martín-Moreno JM, Boyle P, Gorgojo L, Willet WC, et al. Development and validation of a food frequency questionnaire in Spain. Int J Epidemiol 1993;22:512-519.

21. Ordovas JM, Corella D, Demissie S, Cupples, et al. Dietary fat intake determines the effect of a common polymorphism in the hepatic lipase gene promoter on highdensity lipoprotein metabolism: evidence of a strong dose effect in this gene-nutrient interaction in the Framingham Study. Circulation 2002;106:2315-2321.

22. Beynen AC, Katan MB, Van Zutphen LF. Hypo- and hyperresponders: individual differences in the response of serum cholesterol concentration to changes in diet. Adv Lipid Res 1987;22:115-171.

23. Katan MB, Beynen AC. Characteristics of human hypo- and hyperresponders to dietary cholesterol. Am J Epidemiol 1987;125:387-399.

24. Beynen AC, Katan MB. Reproducibility of the variations between humans in the response of serum cholesterol to cessation of egg consumption. Atherosclerosis 1985; 57:19-31.

25. Herron KL, Vega-Lopez S, Conde K, Ramjiganesh T, et al. Men classified as poor hyperresponders to dietary cholesterol feeding exhibit differences in lipoprotein metabolism. J Nutr 2003;133:1036-1042.

26. Garcés C, Benavente M, Lasunción MA, Ortega H, et al. Gender-specific effects of apo E genotype on plasma lipid levels in a population-based sample of 6-7-year-old children in Spain. Acta Paediatr 2002;91:1039-1043.

27. Ostlund RE, Bosner MS, Stenson WF. Cholesterol absorption efficiency declines at moderate dietary doses in normal human subjects. J Lipid Res 1999;40:1453-1458. 